Gorrill, R. H. \& MCNeIL, E. M. (1960). J. gen. Microbiol. 22, 437-442

\title{
The Effect of Cold Diluent on the Viable Count of Pseudomonas pyocyanea
}

\author{
By R. H. GORRILL aNd EVELYN M. McNEIL \\ Department of Bacteriology, Guy's Hospital Medical School, London, S.E. 1
}

SUMMARY : Young actively dividing cultures of Pseudomonas pyocyanea (aeruginosa) at $37^{\circ}$ were killed when diluted into cold liquids which were without effect when used above $18^{\circ}$. In general the simpler the composition of the diluent the more lethal it was; distilled water was the most active. The cooling had to be rapid to be effective. Old cultures were only slightly sensitive to this effect. The killed organisms appeared normal when examined under the microscope. Under similar conditions Staphylococcus aureus was resistant to cold shock.

The killing effect of cold diluent on young Escherichia coli was shown by Sherman \& Albus (1923) who suggested that the organisms passed through a phase of 'rejuvenation' before multiplication. Hegarty \& Weeks (1940) made an extensive investigation into the sensitivity of $\boldsymbol{E}$. coli to cold shock and showed that low osmotic pressure and cold were both necessary in order to kill organisms exposed to these conditions. Overnight broth cultures became sensitive within $20 \mathrm{~min}$. of their addition to a suitable medium, i.e. before the first bacterial division took place. The organisms showed maximum sensitivity during the log phase of growth. No other report of this phenomenon appeared until that of Meynell (1958) who showed, with $E$. coli, that the number of organisms which appeared to be killed by cold shock depended upon the medium on which they were subsequently plated. The importance of the composition of the diluent and the speed of cooling were also emphasized. Cultures which had suffered cold shock showed no decrease in numbers on direct counting and the bacteria looked normal microscopically. The present investigation stemmed from the observation that direct viable counts of young shaken cultures of Pseudomonas pyocyanea (aeruginosa) diverged from those expected from the turbidity of the cultures. This paper reports work on the sensitivity of $\boldsymbol{P}$. pyocyanea to cold shock.

\section{METHODS}

Organisms. Cultures of Pseudomonas pyocyanea obtained from patients and from the National Collection of Type Cultures (NCTC) strains 8505 and 8506 were used. In general, all strains behaved in the same way; only the results obtained with NCTC 8506 are reported here. In comparative tests Escherichia coli NCTC 6064 and K 12 (Pasteur Institute, Paris), Salmonella typhimurium 1404 (Wellcome Foundation, London) and Staphylococcus aureus NCTC 8354 were used. The bacteria were grown in L-shaped optical glass tubes in a shaking machine (Gorrill \& Needs, 1958).

Counts. After samples of cultures had been exposed to the test diluent for the 
required time, samples were diluted in the same diluent at the same temperature and counts made by spreading $0.1 \mathrm{ml}$. with a glass spreader over the surface of an infusion agar plate (Southern Group Laboratory, Park Hospital, London, S.E. 13). Four plates were used at each dilution.

Chilling. All initial dilutions were made by adding $0.1 \mathrm{ml}$. culture to $9.9 \mathrm{ml}$. test diluent in $\frac{1}{2} \mathrm{oz}$. screw-capped bottles, thus bringing the culture to the temperature and osmotic pressure of the diluent virtually instantaneously. Bottles of diluent standing in the refrigerator were found to be at $c .4^{\circ}$, while bottles standing on a cooling tray were at $0^{\circ}$. Care was taken with the cooling tray to ensure that the temperature did not fall below $0^{\circ}$.

Diluents. Glass-distilled water was used throughout, and Analar grade chemicals were used, with the exception of the sucrose (T. Kerfoot, Lancashire). The composition of the buffer was described by Challice \& Gorrill (1954). Ringer's solution (g./l. water: sodium chloride, 9.0; calcium chloride, $0 \cdot 25$; potassium chloride, $\mathbf{0 . 4 2}$ ) was used as the basis for the study of the effect of different ions on the production of cold shock. Cultures were grown overnight in $10 \mathrm{ml}$. digest broth (Southern Group Laboratory) in 1 oz. bottles. Samples $(0.2 \mathrm{ml}$.) of these overnight stocks were added to $9.8 \mathrm{ml}$. digest broth and shaken in a water bath at $37^{\circ}$.

\section{RESULTS}

The initial observation was made on cultures of Pseudomonas pyocyanea diluted in isotonic phosphate buffer at $4^{\circ}$. The true viable count was estimated by making the dilutions in digest broth at $37^{\circ}$. As will be seen later, similar values were obtained when the dilutions were made in distilled water at $37^{\circ}$. The use of distilled water had the advantage of diminishing the amount of frothing during dilution and was used in some experiments. When the effect of cold diluent was studied more closely, it was realized that the composition and temperature of the diluent and the time of exposure all played a part in the final result. In early experiments it was clear that distilled water was more effective than any other diluent in producing cold shock. Therefore, the effect of temperature and time of cooling were studied with distilled water as diluent.

\section{The effect of temperature on killing}

As shown in Fig. 1, temperatures between $37^{\circ}$ and $18^{\circ}$ were without effect; below $4^{\circ}$ the effect was marked. It is difficult to produce controlled temperatures between these two ranges and so the temperature of onset of the effect was not determined. It will be seen that with Pseudomonas pyocyanea, unlike Escherichia coli, overnight cultures were sensitive to cold water. However, the increase in sensitivity that occurred during growth was similar to that already reported.

When the cultures were chilled from $37^{\circ}$ to $0^{\circ}$ during a period of $30-60 \mathrm{~min}$. in distilled water the cold-shock effect was not seen. This, together with the lack of effect by distilled water above $18^{\circ}$, suggests that the phenomenon depends upon the combination of a sudden decrease in temperature and a low salt concentration. 


\section{Effect of time of cooling}

In Fig. 2 are seen the effects of using distilled water at $0^{\circ}$ for different times. The result labelled 'immediate' was produced by using ice-cold water in the normal viable count. This curve has been included to show the very marked decrease in viable count which occurred with the most unfavourable diluent tested in this series in a routine counting system. A decrease in the number of viable organisms continued for some hours, but is not shown in Fig. 2.

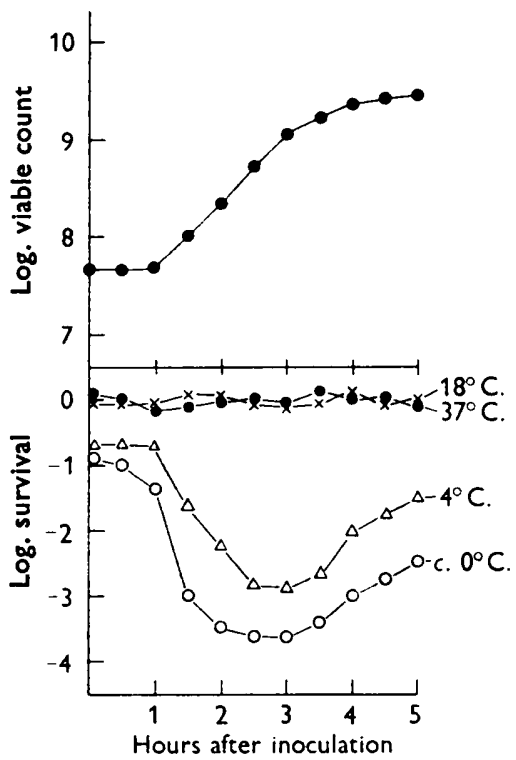

Fig. 1

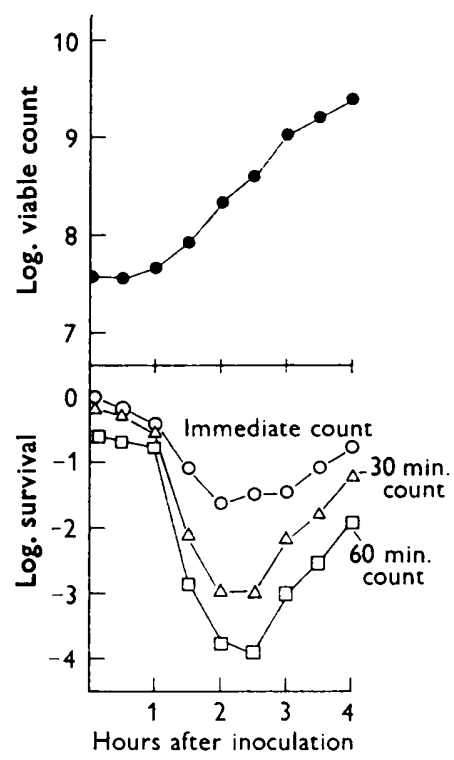

Fig. 2

Fig. 1. The numbers of Pseudomonas pyocyanea surviving for $1 \mathrm{hr}$. in distilled water at different temperatures.

Fig. 2. The effect of time of exposure on survival in distilled water at $0^{\circ}$.

\section{The effect of the composition of the diluent}

An arbitrary period of $1 \mathrm{hr}$. at $0^{\circ}$ was chosen when comparing the effect of the composition of the diluent on its ability to produce cold shock. In general, the more complex the composition of the diluent the less efficient it was as a shocking agent. In order of increasing shocking activity the different diluents tested were: broth, $10 \%(w / v)$ sucrose, buffer, Ringer's solution, $\mathbf{0} \cdot 85 \%(w / v)$ $\mathrm{NaCl}$ ('normal saline').

To study the effect of different ions on the shocking effect of the diluent two groups of experiments were made.

In the first group, tests were made to see how far solutions could be diluted before they produced cold shock. A representative example is given in Fig. 3 which shows that a $1 / 4$ dilution of $0.85 \%$ saline or of Ringer's solution greatly increased their shocking effect. This confirms the results of Meynell (1958) who 
used 1/4 strength Ringer's solution as test diluent. A 1/2 dilution of any of the salt diluents used gave a marked increase in ability to produce cold shock.

In the second group of experiments the effect of individual ions was considered. As seen in Fig. 3, Ringer's solution was less effective in shocking than normal saline; Ringer's solution contains also calcium chloride and potassium chloride. Figure 4 shows that calcium chloride was more effective than potassium chloride when added to normal saline in preventing shocking. Equivalent amounts of other calcium salts were then tested and found to be as effective as calcium chloride. Magnesium ion had an effect intermediate between calcium and potassium. The results may be summarized by saying that divalent ions appeared to be more cffective than univalent ions in decreasing the lethal effect of cold normal saline. Attempts were made to extend these observations to mixtures of three salts, but the differences between most mixtures were less than the error of the counting method, and the experiments were abandoned.
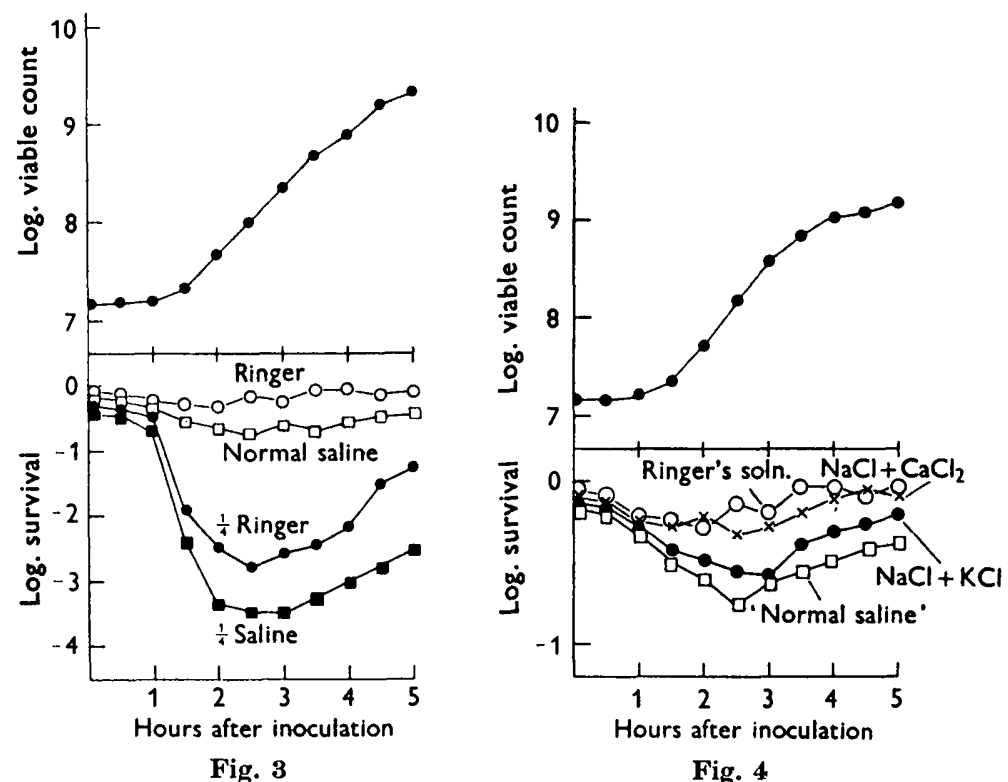

Fig. 4

Fig. 3. The effect of concentration of diluent upon its ability to produce cold shock at $0^{\circ}$ for $1 \mathrm{hr}$.

Fig. 4. The effect of the composition of the diluent on its ability to produce cold shock $\left(0^{\circ} ; 1 \mathrm{hr}.\right)$.

\section{Effect of log-phase growth on sensitivity to cold}

Pseudomonas pyocyanea showed its maximum sensitivity to cold shock during the phase of active bacterial division. While it is usually assumed that these two events are causally related it is possible that organisms passing out of the lag phase lose their sensitivity to cold shock in 3-4 hr. This would appear in the experiments reported above as indicating a sensitivity of log-phase cultures to cold shock which was in fact a coincidence. This possibility may be tested by 
maintaining the culture in the log phase for a longer period. A simple method previously described (Gorrill \& Needs, 1958) was used: once a culture was in the actively dividing phase it was diluted at suitable intervals into fresh warm shaken broth. In this way a series of tubes were set up, the most recent containing actively dividing organisms while the original tube contained organisms in the stationary phase. One such experiment is illustrated in Fig. 5; it will be seen that organisms remained highly sensitive to cold shock as long as they remained in the log phase.

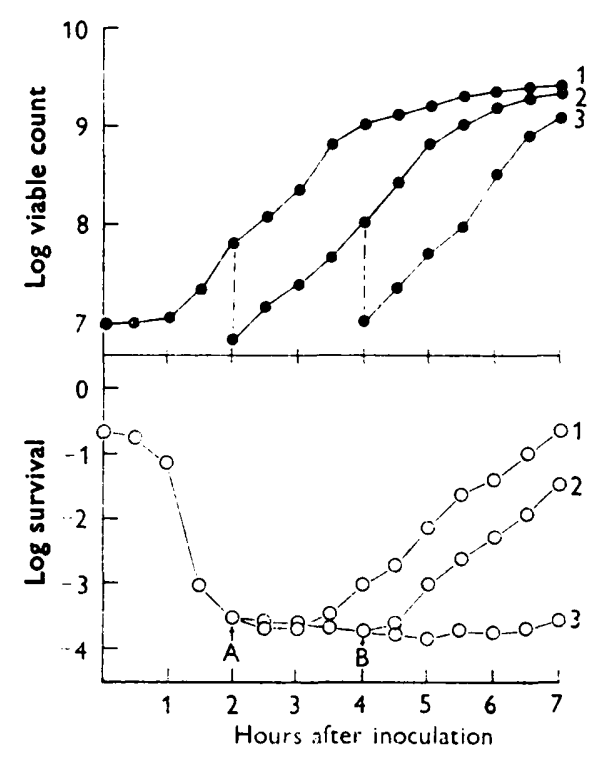

Fig. 5

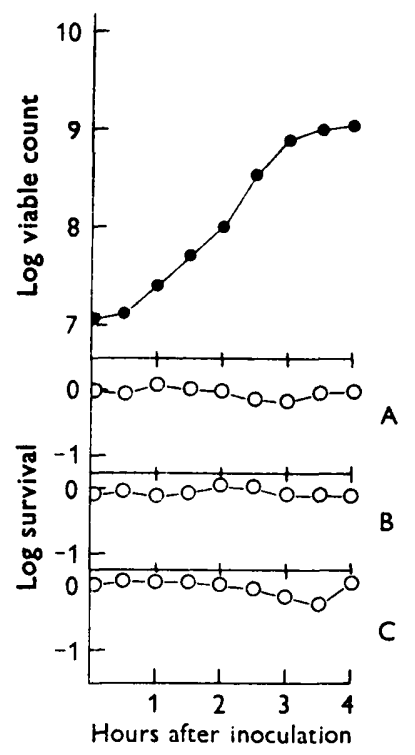

Fig. 6

Fig. 5. The effect of repeated dilution of a $P$.pyocyanea culture on its sensitivity to cold water

$\left(0^{\circ}\right)$ for $1 \mathrm{hr}$. Tube numbers: $1,2,3$. Tube 2 was set up from tube 1 at point $A(2 \mathrm{hr}$.) and tube 3 from tube 2 at point $B(4 \mathrm{hr}$.

Fig. 6. The number of staphylococci surviving for $1 \mathrm{hr}$. at $0^{\circ}$ in: (A) distilled water; (B) normal saline; (C) buffer.

\section{The appearance of shocked cultures}

Cultures which had suffered cold shock to the extent that less than $1 \%$ survived as judged by viable counts were examined by dark-ground and phasecontrast microscopy, and films were stained by methylene blue and examined under the ordinary light microscope; normal control cultures were also examined. No morphological differences as between shocked and unshocked organisms could be distinguished. Total counts were made in a counting chamber under phase-contrast microscopy of chilled and control cultures; the counts did not differ from one another by more than $20 \%$. Considering the inherent errors in the method this suggests that they were similar. These findings confirm those of Meynell (1958) with Escherichia coli. 


\section{Sensitivity to cold shock of other species}

There is very little information about the distribution of sensitivity to cold shock amongst the different species of bacteria. To obtain some information on this point the following organisms were tested: Escherichia coli NCTC 6064 and K 12, Salmonella typhimurium 1404 and Staphylococcus aureus NCTC 8354. The first three were all sensitive to cold shock though less so than Pseudomonas pyocyanea. The Staphylococcus showed a marked resistance to cold shock as is seen in Fig. 6, where the survivals after exposure for $1 \mathrm{hr}$. to ice-cold water, saline and buffer are shown.

\section{DISCUSSION}

The practical effects of cold shock in routine counting procedures may be considerable. With water at $0^{\circ}$ even immediate dilution and counting may kill over $90 \%$ of Pseudomonas pyocyaneas exposed. This is, of course, an extreme case, both with regard to diluent and organism. Staphylococci would show no detectable effect in these conditions. Ice-cold buffer would be without effect on $P$. pyocyanea if it were used for immediate dilution. Hence it is important before using an ice-cold diluent to be certain that it is without effect on the organism being used. When considering the specific effect of different ions in cold-shock it is interesting to contrast the findings of Wilson (1922) who worked at room temperature; Ringer's solution, tap water, saline, in that order, were progressively more deleterious to Salmonella suipestifer. In experiments to determine the minimum concentration of Ringer's solution which would protect, Wilson found this to be about a 1/16 dilution. It will be seen from these findings that the lethal effect of diluents at room temperatures may be quite different from those at refrigerator temperatures.

\section{REFERENCES}

Challice, C. E. \& Gorrill, R. H. (1954). Some observations on the morphological changes in $E$. coli accompanying induction by ultraviolet light. Biochim. biophys. Acta, 14, 482.

Gorrill, R. H. \& Neens, D. A. (1958). A simple apparatus for shaking bacterial cultures. J. clin. Path. 11, 89.

Hegarty, C. P. \& Weeks, O. B. (1940). Sensitivity of Escherichia coli to cold shock during the logarithmic growth phase. J. Bact. 39, 475.

Meynews, G. G. (1958). The effect of sudden chilling on Escherichia coli. J. gen. Microbiol. 19, 380.

Sherman, J. M. \& Aldus, W. R. (1923). Physiological youth in bacteria. J. Bact. 8, 127.

Wrrson, G. S. (1922). The proportion of viable bacteria in young cultures with especiul reference to the technique employed in counting. J. Bact. 7, 405. 